\title{
DETERMINATION OF TOTAL HARMONIC DISTORTION (THD) ON A 33KV DISTRIBUTION NETWORK: A CASE STUDY OF ISLAND BUSINESS DISTRICT
}

\author{
L. M. Adesina ${ }^{1}{ }^{*}$, O. A. Fakolujo ${ }^{2}$

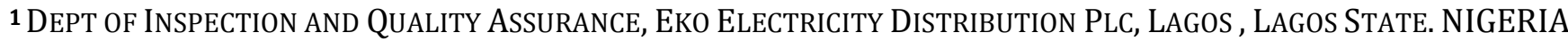 \\ 2 DEPARTMENT OF ELECTRICAL/ELECTRONIC ENGINEERING, UNIVERSITY OF IBADAN, IBADAN. OYO STATE. NIGERIA \\ E-mail addresses: 1 lambe.adesina@ekedc.com, 2 ao.fakolujo@ui.edu.ng
}

\begin{abstract}
Modern day AC power systems are proliferated by the introduction of several kinds of nonlinear loads which generate harmonics in a power system and this has a cumulative negative effect on power quality. Examples of nonlinear loads are power electronic devices, which cause distortion of voltage and current waveforms and consequently create a number of harmonics within the power system. This paper presents the estimation of Total Harmonic Distortion (THD) of the Distribution lines in the 33kV distribution network of Island Business District, Eko Electricity Distribution PIc, taken as a case study using a set of busbar voltage results, obtained from previous studies on power flow and harmonic analyses of each time of the $33 \mathrm{kV}$ feeder restoration. The harmonics and their impedances on each line at the scenario time of the $33 \mathrm{kV}$ feeders restored are presented. The estimated THD of each line and overall network average THD are also presented.
\end{abstract}

Keywords: Power quality, Harmonics, Total Harmonic Distortion, Feeder restoration

\section{INTRODUCTION}

Power system harmonics is not a new phenomenon. In fact, a text published by Steinmetz in 1916, devoted considerable attention to the study of harmonics in 3phase power systems. In Steinmetz's days, the main concern was third harmonic currents, caused by saturated iron in transformers and machines. He was the first to propose Delta connections for blocking third harmonic currents. Steinmetz's contribution and an overall history of harmonics have been published by Owen, E.L [1]. The emergence of power electronic loads in a power system began during the late 80 's and early 90 's. They offer the advantages of efficiency and controllability. However, a downside to this is that they also draw non-sinusoidal currents from AC power systems, and these currents interact negatively with the power system impedances to create voltage harmonics and in some cases, resonance. Studies have shown that harmonic distortion levels in distribution systems are on the rise as power electronic devices continue to proliferate [2]. The main effects of harmonics in power systems are heating, overloading and ageing of equipment, and increased losses.
Besides the increase in the number of harmonic sources, the number of devices sensitive to the harmonic distortion has also increased [3]. Harmonics may lead to malfunctioning of power system components and electronic devices.

The harmonic distortion is one of the indices of power quality of a power system [4]. This is because of large nonlinear loads that have been used. The IEEE Standard 519-1992 provides a solution for the limitation and mitigation of harmonics [4]. The Total Harmonic Distortion (THD) is the common measurement index of harmonic distortion $[5,6]$. THD is applied to both current and voltage and is defined as the root mean square (rms) value of the $h^{\text {th }}$ harmonic voltage, divided by the rms value of the fundamental harmonic voltage, and then multiplied by $100 \%$ as shown in subsequent discussions. THD of current varies from few percent to more than 100\%.THDs of voltage are usually less than $5 \%$ and widely considered to be acceptable, while values above $10 \%$ are definitely unacceptable and will cause problems for sensitive equipment and loads [5]. 


\section{IEEE HARMONIC STANDARDS AND TOTAL HARMONIC DISTORTION}

As far as the impact of harmonics on power system equipment is concerned, it can be generally stated that harmonics cause equipment to be subjected to voltages and currents at frequencies for which it was not designed. The effects of such exposure are usually not instantly visible, but can have serious medium and long term consequences. Some of the practical harmonic related problems encountered by utilities include voltage distortion in distribution feeders, increased RMS currents, which causes overheating and line losses, malfunctioning of solid-state fuses, breakers and relays, tripping of harmonic voltagesensitive equipment [7], interaction of harmonic currents with the capacitances of power factor correction capacitors (generally installed in industrial plants, commercial buildings and fluorescent lightings to improve individual light fittings), which can cause overheating, disrupt, and/or damage plant and equipment [8], etc.

Furthermore, Harmonic currents are injected from harmonic producing loads into the utility network in radial distribution feeders. These harmonic currents affect the operation of other electrical and electronic equipment connected on the same distribution feeder, including the harmonic producing loads. Deeper into the utility distribution and transmission system, it becomes difficult to discern the direction of harmonic power flow, partly due to the highly interconnected and meshed nature of such systems, and partly due to the impact on voltage support capacitors used by the utility and its customers. As utilities and customers change their connected loads, system impedance also changes, resulting into resonances and harmonic problems which were initially none existent [9]. Such problems are not easily identified without a detailed system analysis [10, 11]. An important aspect of power quality monitoring is the collection of information regarding the performance of the system in terms of voltage and current harmonics. For voltage and current harmonics, the obtained information (magnitude and phase) are usually compared with standards like IEEE 519 harmonic standards to evaluate the influence of these events.

\subsection{IEEE 519-1992 Harmonic Standard}

IEEE 519 was initially introduced in 1981 as an "IEEE Guide for Harmonic Control and Reactive Compensation of Static Power Converters". It originally established levels of voltage distortion acceptable to the distribution system for individual non-linear loads. With the rising increase usage of industrial non-linear loads, such as variable frequency drives, it became necessary to revise the standard.

In USA, IEEE applies whereas in Europe, a different standard (IEC) applies. Measured harmonics significantly higher than the recommended levels are considered unacceptable. All of the standards make use of the total harmonic distortion (THD) voltage or current, defined as [12]:

$$
T H D=\frac{100 \sqrt{\sum_{h=2}^{k} U_{h r m s}^{2}}}{U_{1 r m s}}
$$

Where $U_{1 r m s}$ and $U_{\text {hrms }}$ are the fundamental and $h^{\text {th }}$ (higher order) harmonic voltage components respectively.

The main standards are the followings (9):

IEEE 519: this standard sets limits for percentage "individual harmonic component distortion" and "THD". It limits both utility voltage and end user current distortion at the point of common coupling.

a) IEC 1000-2-4 (1994): it prescribes the compatibility levels for industrial and non-public networks. It applies to low and medium voltage supplies.

b) IEC 1000-1-1 (1992): Definitions used in IEC 1000

c) IEC 1000-2-2 (1990): It defines the compatibility levels for individual harmonic voltages in public low voltage systems.

The IEEE 519-1992 standard established recommended guidelines for harmonic voltages on the utility distribution system as well as harmonic currents within the industrial distribution system [8]. According to the standard, the industrial system is responsible for controlling the harmonic currents created in the industrial workplace. Since harmonic currents reflected through distribution system impedances generate harmonic voltages on the utility distribution systems, the standard proposes guidelines based on industrial distribution system design. IEEE 519-1992 defines levels of harmonic currents and voltages that an industrial user can inject onto the utility distribution system [13-16].

\subsection{Total Harmonic Distortion (THD)}

Although the harmonic content of a power system may be quite small relative to the fundamental in most circumstances, for exactness the rms value of a current or voltage waveform requires the harmonic content to be considered such that: 


$$
U_{r m s}=\sqrt{\sum_{h=1}^{\infty}\left(\frac{1}{\sqrt{2}} U_{h}\right)^{2}}
$$

Where $U_{\text {rms }}$ and $U_{h}$ are the rms value and peak $h^{\text {th }}$ harmonic component of voltage or current.

The rms voltage or current can also be used to quantify the level of distortion of the waveform. The total harmonic distortion of voltage or current waveform $\left(\mathrm{THD}_{\mathrm{U}}\right)$ is calculated using equation (3):

$$
T H D_{U}=\sqrt{\sum_{h=2}^{\infty}\left(U_{h}\right)^{2}}=\sqrt{\left(\frac{U_{r m s}}{U_{1 r m s}}\right)^{2}-1}
$$

Where $\mathrm{THD}_{\mathrm{U}}$ represents voltage or current total harmonic distortion (alternatively represented as $\mathrm{THD}_{\mathrm{V}}$ and $\mathrm{THD}_{\mathrm{I}}$ respectively) and $\mathrm{U}_{1 \mathrm{rms}}$ is the rms fundamental voltage or current. Alternatively rms voltage or current can be represented in terms of total harmonic distortion:

$$
U_{r m s}=\sqrt{\sum_{h=1}^{\infty} U_{h r m s}^{2}}=U_{1 r m s} \sqrt{1+T H D_{U}^{2}}
$$

As distribution system fundamental voltage and current rarely remain static in magnitude at different times throughout the day, the definition for total harmonic distortion may at times provide a misleading value for the harmonic distortion level. This is especially true for distribution system fundamental currents that fall close to zero at certain periods of the day, resulting in large values of $\mathrm{THD}_{\mathrm{I}}$. For this reason a modified index for harmonic distortion may be used with the harmonic content of the waveform expressed as a percentage of a fixed nominal value rather than the fundamental value, giving total demand distortion $\left(\mathrm{TDD}_{\mathrm{U}}\right)$ [12]:

$$
T D D_{u}=\frac{1}{U_{\text {nom }}} \sqrt{\sum_{h=2}^{\infty}\left(\frac{1}{\sqrt{2}} U_{h}\right)^{2}}
$$

The fixed value $U_{\text {nom }}$ is required to be specified and may be a maximum rms value, maximum demand, average or selected nominal system value.

Generally, one of the most important tools for understanding and analysing of harmonics as well as for the standardization work is harmonic measurements. The estimation of harmonics is of high importance for efficiency of the power system network [17]. Harmonic measurements are also important for grid companies and end users to characterize the performance of their networks and develop solutions to harmonic problems.

\section{33KV NETWORK OF ISLAND BUSINESS DISTRICT OF EKO ELECTRICITY DISTRIBUTION PLC (EKEDP): CASE STUDY}

The success of a power system harmonic study depends critically on the data used to model power system components. Consequently, this paper makes use of the Busbar voltage solutions obtained from a power flow study and harmonic analysis results of scenario hours of feeder restoration per day, on $33 \mathrm{kV}$ network of Island Business District (IBD) of EKEDP shown in Figure 1. The results obtained from the research work were used as the source data for the analysis of network THD, presented in this paper. Figure 2, however, shows the line model of the distribution network used earlier on in obtaining the harmonics on various lines of the network.

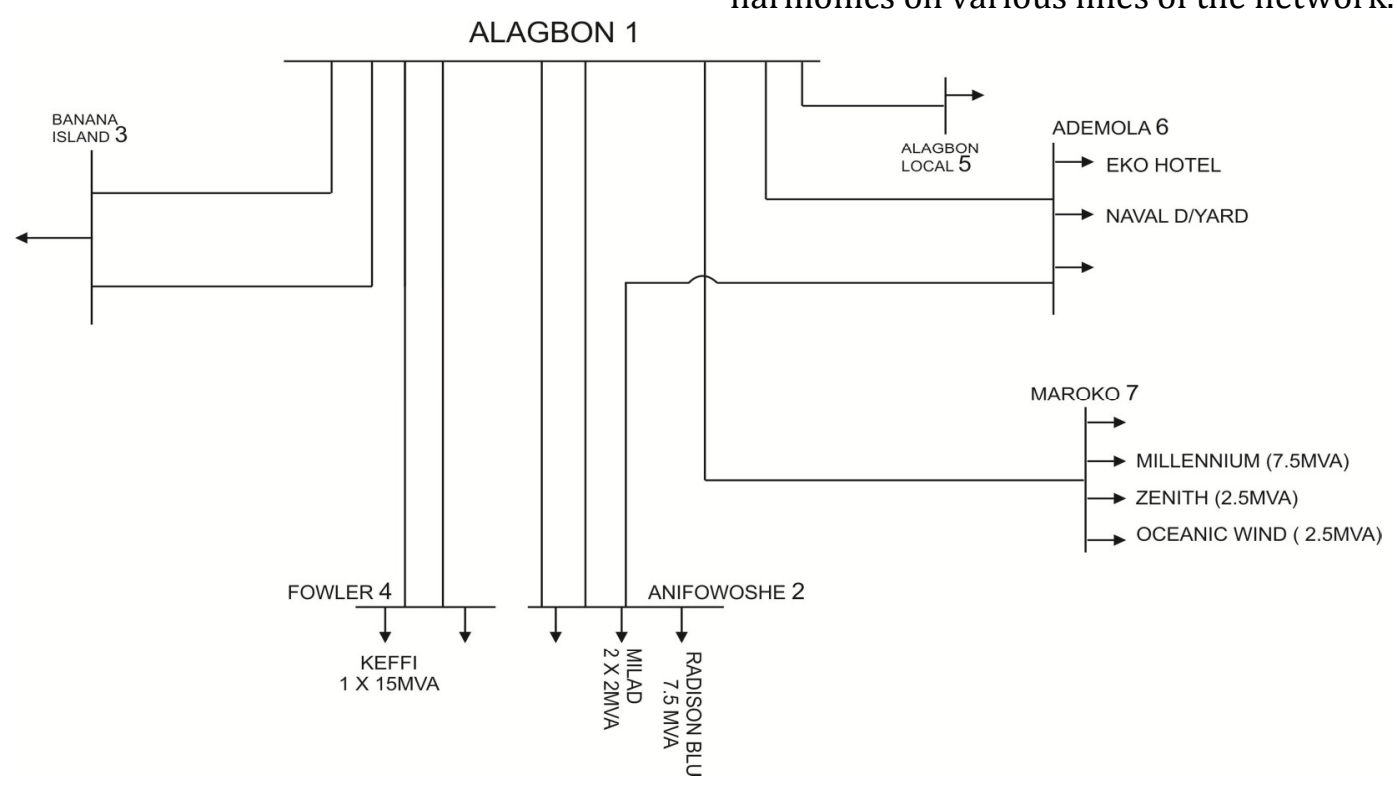

Figure 1: 33kV Network of Islands Business Unit, EKEDP 


\section{Eontinuous}

powergul

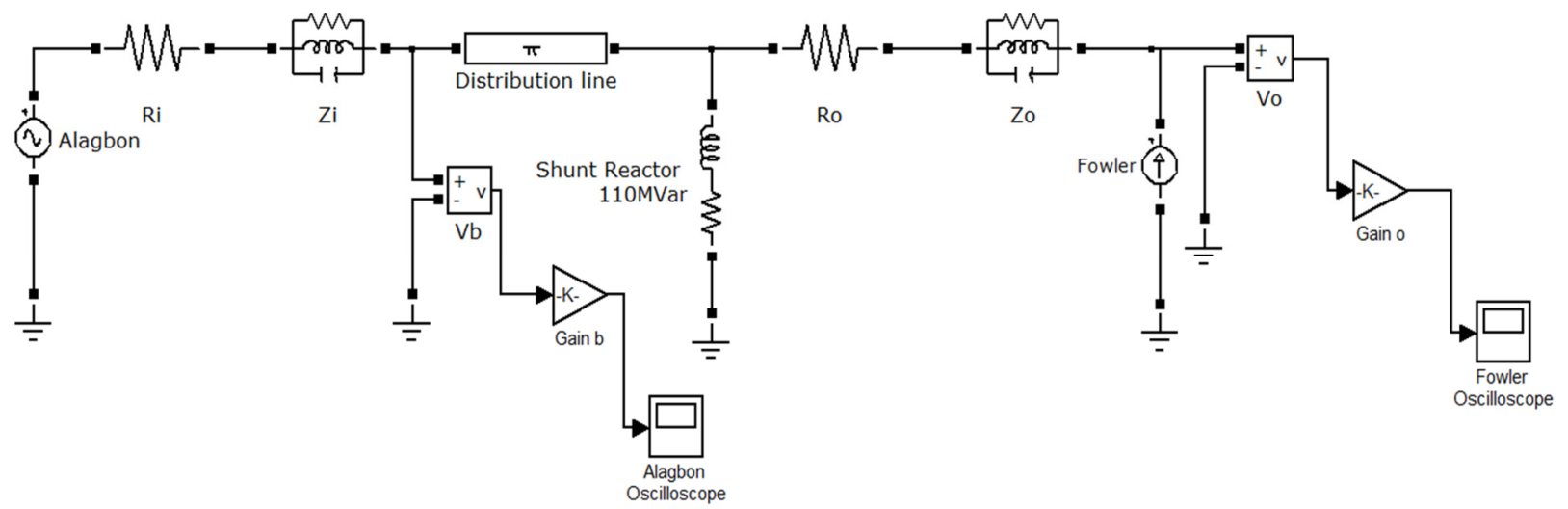

Figure 2: Formulated Model of Distribution Line Alagbon - Fowler Busbars as example

The source data were obtained from the real life simulations using the MATLAB/Simulink platform and the summaries of the findings/observations from the simulations are given in Tables 1 to 4, which show the distribution line harmonics and their Impedances at a frequency range of $1500 \mathrm{~Hz}$, for all the cases of scenario hours of the $33 \mathrm{kV}$ feeder's restoration in the network of case study. However, the seven distribution lines, within the $33 \mathrm{kV}$ distribution network of IBD considered during simulation are as follows:

1. Alagbon (ALG) - Ademola (ADM)

2. Alagbon (ALG) - Alagbon Local (ALG/L)

3. Alagbon (ALG) - Anifowoshe (ANI)

4. Alagbon (ALG) - Fowler (FOW)
5. Ademola (ADM) - Maroko (MAR)

6. Ademola (ADM) - Anifowoshe (ANI)

7. Alagbon (Alagbon) - Banana Island (BAN/I)

During simulation, 10 cascaded $\pi$-networks were used in order to observe all harmonics present on each of the lines. The reason for selecting the $\pi$-network topology for the simulation was because the longest of the Distribution lines within the $33 \mathrm{kV}$ network is $6.84 \mathrm{~km}$. The harmonic impedance values given in tables 1 to 4 are extracted from the harmonic Impedance / Frequency plots results of the harmonic analysis of the same network as shown in [18].

At 02:00Hrs

Case 1: ALG - ALG/L

Case 2: ALG - FOW Distribution Line

Table 1: Distribution Line Harmonics and their Impedance for cases of 02:00Hrs

\begin{tabular}{lccccc}
\hline Distribution Line & Length $(\mathrm{km})$ & $\begin{array}{c}\text { Sending Bus Voltage } \\
(\mathrm{kV})\end{array}$ & $\begin{array}{c}\text { Receiving Bus Voltage } \\
(\mathrm{kV})\end{array}$ & Harmonics & $\begin{array}{c}\text { Harmonic Impedance } \\
(\Omega)\end{array}$ \\
\hline ALG-ADM & 6.13 & 33 & 0 & - & - \\
ALG-ALG/L & 0.15 & 33 & 32.997 & $2^{\text {nd }}$ & $10^{1.881}$ \\
ALG-ANI & 6.84 & 33 & 0 & - & - \\
ALG-FOW & 3.00 & 33 & 32.881 & $2^{\text {nd }}, 6^{\text {th }}, 15^{\text {th }}, 24^{\text {th }}$ & $10^{2.0}, 10^{2.51}$ \\
ADM-MAR & 1.80 & 0 & 0 & - & $10^{2.27}, 10^{2.2}$ \\
ADM-ANI & 2.40 & 0 & 0 & - & - \\
ALG-BAN/I & 5.00 & 33 & 0 & - & - \\
\hline
\end{tabular}

At 09:00Hrs

Case 1: ALG - ADM Distribution Line

Case 2: ALG -ALG/L Distribution Line

Case 3: ALG - ANI Distribution Line

Case 4: ALG - FOW Distribution Line 
Case 5: ADM - ANI Distribution Line

Case 6: ALG -BAN/I Distribution Line

Table 2: Distribution Line Harmonics and their Impedance for cases of 09:00Hrs

\begin{tabular}{|c|c|c|c|c|c|}
\hline Distribution Line & $\begin{array}{l}\text { Length } \\
(\mathrm{km})\end{array}$ & $\begin{array}{l}\text { Sending Bus } \\
\text { Voltage }(\mathrm{kV})\end{array}$ & $\begin{array}{l}\text { Receiving Bus } \\
\text { Voltage }(\mathrm{kV})\end{array}$ & Harmonics & $\begin{array}{l}\text { Harmonic Impedance } \\
(\Omega)\end{array}$ \\
\hline ALG-ADM & 6.13 & 33 & 32.711 & $\begin{array}{l}2^{\text {td }}, 3^{\text {rd }}, 8^{\text {th }}, 12^{\text {th }}, 16^{\text {th }}, 20^{\text {th }} \\
23^{\text {rd }}, 26^{\text {th }}, 28^{\text {th }}, 29 \text { th }\end{array}$ & $\begin{array}{l}10^{2.01}, 10^{2.65}, 10^{2.28}, 10^{2.15}, \\
10^{2.05}, 10^{2.01}, 10^{1.56}, 10^{1.95}, \\
10^{1.86}, 10^{1.74}\end{array}$ \\
\hline ALG-ALG/L & 0.15 & 33 & 32.997 & $2^{\text {nd }}$ & $10^{2.05}$ \\
\hline ALG-ANI & 6.84 & 33 & 32.645 & $\begin{array}{l}2^{\text {nd }}, 3^{\text {rd }}, 7^{\text {th }}, 11^{\text {th }}, 14^{\text {th }}, 18^{\text {th }}, \\
21^{\text {st }}, 23^{\text {rd }}, 25^{\text {th }}, 26^{\text {th }}, 27^{\text {th }}\end{array}$ & $\begin{array}{l}10^{2.12}, 10^{2.65}, 10^{2.30}, 10^{2.14}, \\
10^{2.06}, 10^{1.97}, 10^{1.91} 10^{1.84}, \\
10^{1.69}, 10^{1.49}, 10^{0.99}\end{array}$ \\
\hline ALG-FOW & 3.00 & 33 & 32.860 & $2^{\text {nd }}, 6^{\text {th }}, 15^{\text {th }}, 24^{\text {th }}$ & $10^{1.99}, 10^{2.51}, 10^{2.27}, 10^{2.20}$ \\
\hline ADM-MAR & 1.80 & 32.711 & 0 & - & - \\
\hline ADM-ANI & 2.40 & 32.711 & 32.645 & $2^{\text {nd }}, 8^{\text {th }}, 19^{\text {th }}$ & $10^{1.98}, 10^{2.47}, 10^{2.28}$ \\
\hline ALG-BAN/I & 5.00 & 33 & 32.942 & $\begin{array}{l}2^{\text {nd }}, 3^{\text {rd }}, 9^{\text {th }}, 15^{\text {th }}, 20^{\text {th }}, 24^{\text {th }}, \\
29 \text { th }\end{array}$ & $\begin{array}{l}10^{1.96}, 10^{2.61}, 10^{2.28}, 10^{2.15}, \\
10^{2.08}, 10^{2.05}, 10^{2.01}\end{array}$ \\
\hline
\end{tabular}

At 21:00Hrs

Case 1: ALG - ADM Distribution Line

Case 2: ALG - ANI Distribution Line

Case 3: ALG - FOW Distribution Line

Case 4: ADM - ANI Distribution Line

Table 3: Distribution Line Harmonics and their Impedance for Cases of 21:00Hrs

\begin{tabular}{|c|c|c|c|c|c|}
\hline Distribution Line & Length $(\mathrm{km})$ & $\begin{array}{l}\text { Sending Bus } \\
\text { Voltage }(\mathrm{kV})\end{array}$ & $\begin{array}{l}\text { Receiving Bus } \\
\text { Voltage }(\mathrm{kV})\end{array}$ & Harmonics & Harmonic Impedance $(\Omega)$ \\
\hline ALG-ADM & 6.13 & 33 & 32.83 & $\begin{array}{c}2^{\text {nd }}, 3^{\text {rd }}, 8^{\text {th }}, 12^{\text {th }}, 16^{\text {th }}, \\
20^{\text {th }}, 23^{\text {rd }}, 26^{\text {th }}, 28^{\text {th }}, \\
29^{\text {th }}\end{array}$ & $\begin{array}{c}10^{2.01}, 10^{2.64}, 10^{2.27}, 10^{2.15} \\
10^{2.05}, 10^{2.01}, 10^{1.93}, 10^{1.86} \\
10^{1.74}, 10^{1.56}\end{array}$ \\
\hline ALG-ALG/L & 0.15 & 33 & 0 & - & - \\
\hline ALG-ANI & 6.84 & 33 & 32.73 & $\begin{array}{l}2^{\text {nd }}, 3^{\text {rd }}, 7^{\text {th }}, 11^{\text {th }}, 14^{\text {th }}, \\
18^{\text {th }}, 21^{\text {st }}, 23^{\text {rd }}, 25^{\text {th }}, \\
26^{\text {th }}, 27^{\text {th }}\end{array}$ & $\begin{array}{c}10^{2.12}, 10^{2.66}, 10^{2.37}, 10^{2.14} \\
10^{2.06}, 10^{1.97}, 10^{1.91}, 10^{1.84} \\
10^{1.69}, 10^{1.49}, 10^{0.99}\end{array}$ \\
\hline ALG-FOW & 3.00 & 33 & 32.87 & $2^{\text {nd }}, 6^{\text {th }}, 15^{\text {th }}, 24^{\text {th }}$ & $10^{1.99}, 10^{2.51}, 10^{2.27}, 10^{2.20}$ \\
\hline ADM-MAR & 1.80 & 32.83 & 0 & - & - \\
\hline ADM-ANI & 2.40 & 32.83 & 32.73 & $2^{\text {nd }}, 8^{\text {th }}, 19^{\text {th }}$ & $10^{1.98}, 10^{2.47}, 10^{2.28}$ \\
\hline ALG-BAN/I & 5.00 & 33 & 0 & - & - \\
\hline
\end{tabular}

At 23:00Hrs

Case 1: ALG - ANI Distribution Line

Table 4: Distribution Line Harmonics and their Impedance for Cases of 23:00Hrs

\begin{tabular}{|c|c|c|c|c|c|}
\hline $\begin{array}{l}\text { Distribution } \\
\text { Line }\end{array}$ & $\begin{array}{l}\text { Length } \\
(\mathrm{km})\end{array}$ & $\begin{array}{c}\text { Sending Bus } \\
\text { Voltage } \\
(\mathrm{kV})\end{array}$ & $\begin{array}{c}\text { Receiving Bus } \\
\text { Voltage } \\
(\mathrm{kV})\end{array}$ & Harmonics & $\begin{array}{l}\text { Harmonic Impedance } \\
(\Omega)\end{array}$ \\
\hline ALG-ADM & 6.13 & 33 & 0 & - & - \\
\hline ALG-ALG/L & 0.15 & 33 & 0 & - & - \\
\hline ALG-ANI & 6.84 & 33 & 32.695 & $\begin{array}{l}2^{\text {nd }}, 3^{\text {rd }}, 7^{\text {th }}, 11^{\text {th }}, 14^{\text {th }}, 18^{\text {th }}, \\
21^{\text {st }}, 23^{\text {rd }}, 25^{\text {th }}, 26^{\text {th }}, 27^{\text {th }}\end{array}$ & $\begin{array}{c}10^{2.12}, 10^{2.65}, 10^{2.30}, 10^{2.14}, 10^{2.06}, 10^{1.97} \\
10^{1.91}, 10^{1.84}, 10^{1.69}, 10^{1.49}, 10^{0.99}\end{array}$ \\
\hline ALG-FOW & 3.00 & 33 & 0 & - & - \\
\hline ADM-MAR & 1.80 & 32.83 & 0 & - & - \\
\hline ADM-ANI & 2.40 & 32.83 & 0 & - & - \\
\hline ALG-BAN/I & 5.00 & 33 & 0 & - & - \\
\hline
\end{tabular}




\section{ESTIMATION OF THD VOLTAGE AND CASCADED HARMONIC VOLTAGE $\left(\mathrm{U}_{\mathrm{H}}\right)$ ON THE DISTRIBUTION LINES}

Total Harmonic Distortion Voltage (THDU) of each Distribution line, where the $33 \mathrm{kV}$ feeder is restored, is estimated using the following parameters:

1. The harmonic impedances of the existing harmonics.

2. The exact value of the receiving end voltage of the Distribution line.

3. Active power in MW at the receiving end Busbar.

4. Number of cascaded Distribution lines used during MATLAB/Simulink simulation. In this case, 10 no. were used.

5. Power factor of 0.85

Based on these parameters, Cascaded Harmonic Voltages, $U_{h}$ were obtained for each harmonic generated or involved using equation (6), while $\mathrm{THD}_{\mathrm{u}}$ is then estimated using equation (7).

$$
\begin{gathered}
\mathrm{U}_{\mathrm{h}}=\sqrt{\frac{\text { Power }(p) \times \text { Harmonic Impedance }(Z)}{\sqrt{3} \operatorname{Cos} \emptyset}} \\
\operatorname{THD}_{u}=\frac{1}{N_{c} U_{1}} \sqrt{\sum_{h=2}^{n} U_{h}^{2}}
\end{gathered}
$$

This $\mathrm{THD}_{\mathrm{u}}$ estimation is repeated for all cases under a particular Distribution line. Table 5 shows harmonic frequencies, Cascaded Harmonic Voltage, $\mathrm{U}_{\mathrm{h}}$, as well as estimated THD voltage, $\left(\mathrm{THD}_{\mathrm{u}}\right)$ presented under hours of feeder restoration.

Table 5: Cascaded Harmonic Voltages for each Harmonic Generated on different distribution lines at different

\begin{tabular}{|c|c|c|c|}
\hline$h^{\text {th }}$ harmonic & $\begin{array}{l}\text { Harmonic } \\
\text { Freq. }(\mathrm{Hz})\end{array}$ & $U_{h}(V)$ & $U^{2}{ }_{h}(V)$ \\
\hline \multicolumn{4}{|c|}{$02: 00 \mathrm{Hrs}$} \\
\hline \multicolumn{4}{|c|}{$\begin{array}{l}\text { Case 1: ALG-ALG/L Distribution Line, } \mathrm{V}_{\text {Alagbon Local }}= \\
32.999 \mathrm{kV}, \text { Number of Cascaded Lines, } \mathrm{N}_{\mathrm{C}}=10, \mathrm{P}_{\text {Alagbon }} \\
\text { Local }=4.5 \mathrm{MW} \text {, Power Factor }=0.85, \mathrm{THD}_{\mathrm{u}}=5.26 \% \text {, } \\
\mathrm{U}_{\mathrm{h}}=18.56\end{array}$} \\
\hline \multicolumn{4}{|c|}{ Case 2: ALG - FOW Distribution Line } \\
\hline \multicolumn{4}{|c|}{$\overline{V_{\text {Fowler }}}=32.881 \mathrm{kV}, \mathrm{P}_{\text {Fowler }}=8.7 \mathrm{MW}, \mathrm{THD}_{\mathrm{u}}=20.4 \%$} \\
\hline $2^{\text {nd }}$ & 100 & 23.89 & 570.73 \\
\hline $6^{\text {th }}$ & 300 & 43.63 & 1903.58 \\
\hline $15^{\text {th }}$ & 750 & 33.10 & $1,095.61$ \\
\hline \multirow[t]{2}{*}{$24^{\text {th }}$} & 1200 & 30.54 & 932.42 \\
\hline & & & 4502.34 \\
\hline \multicolumn{4}{|c|}{ 09:00 Hrs } \\
\hline \multicolumn{4}{|c|}{$\begin{array}{l}\text { Case 1: ALG - ADM Distribution Line, } \mathrm{V}_{\text {Ademola }} \text { is } 32.711 \mathrm{kV} \text {, } \\
\mathrm{P}_{\text {Ademola }} \text { is } 10.6 \mathrm{MW}, \mathrm{THD}_{\mathrm{u}}=30 \%\end{array}$} \\
\hline $2^{\text {nd }}$ & 100 & 27.05 & 731.70 \\
\hline
\end{tabular}
times.

Case 2: ALG - ALG/L Distribution Line, $2^{\text {nd }}$ Harmonic is $100 \mathrm{~Hz}, \mathrm{~V}_{\text {Alagbon Local }}$ is $32.997 \mathrm{kV}$ and $\mathrm{P}_{\text {AlagbonLocal }}$ is $3.6 \mathrm{MW}$, $\mathrm{U}_{\mathrm{h}}=16.60 \mathrm{kV}$ and $\mathrm{THD}_{\mathrm{u}}=5.03 \%$

Case 3: ALG - ANI Distribution Line

$\mathrm{V}_{\text {Anifowoshe }}$ is $32.645 \mathrm{kV}, \mathrm{P}_{\text {Anifowoshe }}$ is $11.7 \mathrm{MW}, \mathrm{THD}_{\mathrm{u}}=$

\begin{tabular}{|c|c|c|c|}
\hline \\
\hline $2^{\text {nd }}$ & 100 & 32.22 & 1038.19 \\
\hline $3^{\mathrm{rd}}$ & 150 & 59.59 & 3550.41 \\
\hline $7^{\text {th }}$ & 350 & 39.73 & 1578.62 \\
\hline $11^{\text {th }}$ & 550 & 33.24 & 1104.79 \\
\hline $14^{\text {th }}$ & 700 & 30.11 & 906.31 \\
\hline $18^{\text {th }}$ & 900 & 27.33 & 746.92 \\
\hline $21^{\text {st }}$ & 1050 & 25.54 & 652.05 \\
\hline $23^{\text {rd }}$ & 1150 & 23.48 & 551.16 \\
\hline $25^{\text {th }}$ & 1250 & 19.73 & 389.29 \\
\hline $26^{\text {th }}$ & 1300 & 15.67 & 245.63 \\
\hline \multirow[t]{2}{*}{$27^{\text {th }}$} & 1350 & 8.85 & 78.39 \\
\hline & & & 10841.76 \\
\hline \multicolumn{4}{|c|}{$\begin{array}{l}\text { Case 4: ALG - FOW Distribution Line, } \mathrm{V}_{\text {Fowler }} \text { is } 32.868 \mathrm{kV} \text {, } \\
\mathrm{P}_{\text {Fowler }} \text { is } 9.6 \mathrm{MW}, \mathrm{THD}_{\mathrm{u}}=21.4 \%\end{array}$} \\
\hline $2^{\text {nd }}$ & 100 & 25.10 & 630.03 \\
\hline $6^{\text {th }}$ & 300 & 45.83 & 2100.70 \\
\hline $15^{\text {th }}$ & 750 & 34.77 & 1208.82 \\
\hline \multirow[t]{2}{*}{$24^{\text {th }}$} & 1200 & 32.08 & 1028.88 \\
\hline & & & 4968.43 \\
\hline \multicolumn{4}{|c|}{$\begin{array}{l}\text { Case 5: ADM - ANI Distribution Line, } \mathrm{V}_{\text {Anifowoshe }} \text { is } \\
32.645 \mathrm{kV}, \mathrm{P}_{\text {Anifowoshe }} \text { is } 11.7 \mathrm{MW}, \mathrm{THD}_{\mathrm{u}}=20.7 \%\end{array}$} \\
\hline $2^{\text {nd }}$ & 100 & 27.65 & 764.32 \\
\hline $8^{\text {th }}$ & 400 & 48.32 & 2335.00 \\
\hline \multirow[t]{2}{*}{$19^{\text {th }}$} & 950 & 38.69 & 1497.19 \\
\hline & & & 4596.51 \\
\hline \multicolumn{4}{|c|}{$\begin{array}{l}\text { Case 6: ALG - BAN/I Distribution LineV } V_{\text {Banana Island is }} \\
32.942 \mathrm{kV}, \mathrm{P}_{\text {Banana Island }} \text { is } 3.0 \mathrm{MW}, \mathrm{THD}_{\mathrm{u}}=14.8 \%\end{array}$} \\
\hline $2^{\text {nd }}$ & 100 & 13.66 & 186.73 \\
\hline $4^{\text {th }}$ & 200 & 28.81 & 830.26 \\
\hline $9^{\text {th }}$ & 450 & 19.62 & 384.78 \\
\hline $15^{\text {th }}$ & 750 & 17.00 & 289.21 \\
\hline $20^{\text {th }}$ & 1000 & 15.71 & 246.73 \\
\hline $24^{\text {th }}$ & 1200 & 15.09 & 227.62 \\
\hline \multirow[t]{2}{*}{$29^{\text {th }}$} & 1450 & 14.39 & 207.12 \\
\hline & & & 2372.45 \\
\hline \multicolumn{4}{|c|}{ 21:00 Hrs } \\
\hline \multicolumn{4}{|c|}{$\begin{array}{l}\text { Case 1: ALG - ADM Distribution Line, } \mathrm{V}_{\text {Ademola }} \text { is } \\
32.825 \mathrm{kV}, \mathrm{P}_{\text {Ademola }} \text { is } 6.7 \mathrm{MW}, \mathrm{THD}_{\mathrm{u}}=23.77 \%\end{array}$} \\
\hline $2^{\text {nd }}$ & 100 & 21.53 & 463.63 \\
\hline $3^{\mathrm{rd}}$ & 150 & 44.78 & 2005.24 \\
\hline $8^{\text {th }}$ & 400 & 29.25 & 855.40 \\
\hline
\end{tabular}
$31.9 \%$ 


\begin{tabular}{|c|c|c|c|}
\hline$h^{\text {th }}$ harmonic & $\begin{array}{c}\text { Harmonic } \\
\text { Freq. }(\mathrm{Hz})\end{array}$ & $U_{h}(\mathrm{~V})$ & $U^{2}(\mathrm{~V})$ \\
\hline $12^{\text {th }}$ & 600 & 25.33 & 641.46 \\
\hline $16^{\text {th }}$ & 800 & 22.55 & 508.35 \\
$20^{\text {th }}$ & 1000 & 21.66 & 468.99 \\
$23^{\text {rd }}$ & 1150 & 19.98 & 399.18 \\
$26^{\text {th }}$ & 1300 & 18.18 & 330.50 \\
$28^{\text {th }}$ & 1400 & 15.87 & 251.86 \\
$29^{\text {th }}$ & 1450 & 12.80 & 163.74 \\
\hline & & & 6088.35 \\
\hline
\end{tabular}

Case 2: ALG ANI Distribution Line, $\mathrm{V}_{\text {Anifowoshe }}$ is $32.725 \mathrm{kV}$, $\mathrm{P}_{\text {Anifowoshe }}$ is $9.3 \mathrm{MW}, \mathrm{THD}_{\mathrm{u}}=28.4 \%$

\begin{tabular}{|c|c|c|c|}
\hline & & & \\
\hline $2^{110}$ & 100 & 28.76 & 827.13 \\
\hline $3^{\text {rd }}$ & 150 & 53.43 & 2854.79 \\
\hline $7^{\text {th }}$ & 350 & 35.38 & 1251.92 \\
\hline $11^{\text {th }}$ & 550 & 29.60 & 876.14 \\
\hline $14^{\text {th }}$ & 700 & 26.84 & 720.40 \\
\hline $18^{\text {th }}$ & 900 & 24.39 & 595.08 \\
\hline $21^{\text {st }}$ & 1050 & 22.74 & 517.10 \\
\hline $23^{\mathrm{rd}}$ & 1150 & 20.91 & 437.09 \\
\hline $25^{\text {th }}$ & 1250 & 17.63 & 310.87 \\
\hline $26^{\text {th }}$ & 1300 & 13.99 & 195.69 \\
\hline $27^{\text {th }}$ & 1350 & 07.87 & 61.88 \\
\hline & & & 8648.09 \\
\hline $\begin{array}{l}\text { Case 3: ALC } \\
\mathrm{P}_{\text {Fowler }} \text { is } 9.7\end{array}$ & $\begin{array}{l}\text { stribut } \\
u=18\end{array}$ & le, $V_{\text {Fowl }}$ & $2.867 \mathrm{kV}$ \\
\hline $2^{\text {nd }}$ & 100 & 24.62 & 606.14 \\
\hline $8^{\text {th }}$ & 400 & 43.18 & 1864.55 \\
\hline $19^{\text {th }}$ & 950 & 34.50 & 1190.08 \\
\hline & & & 3660.77 \\
\hline & 23: & & \\
\hline $\begin{array}{l}\text { Case 1: ALC } \\
32.695 \mathrm{kV},\end{array}$ & $\begin{array}{l}\text { tributi } \\
10.20\end{array}$ & $\begin{array}{l}\text { e, } V_{\text {Anifon }} \\
H^{\prime} D_{u}=\end{array}$ & \\
\hline 2nd & 100 & 30.22 & 913.25 \\
\hline $3 r d$ & 150 & 55.64 & 3095.81 \\
\hline 7 th & 350 & 37.18 & 1382.35 \\
\hline 11th & 550 & 30.93 & 956.66 \\
\hline 14 th & 700 & 23.21 & 795.80 \\
\hline 18th & 900 & 25.42 & 646.18 \\
\hline 21th & 1050 & 23.73 & 563.11 \\
\hline 23th & 1150 & 21.89 & 479.17 \\
\hline 25th & 1250 & 18.42 & 339.30 \\
\hline 26th & 1300 & 14.63 & 214.04 \\
\hline 27 th & 1350 & 08.23 & 67.73 \\
\hline & & & 9453.41 \\
\hline
\end{tabular}

\section{NETWORK AVERAGE THD}

For the determination of the network average $\mathrm{THD}_{\mathrm{u}}$, (equation 8) is used and the respective values obtained for each of the $\mathrm{D} / \mathrm{Ls}$ in the network is shown in Table 6.

Average $T H D_{u}=\sum_{n=1}^{k}\left(T H D_{u}\right)_{n}$

\section{DISCUSSION}

From the result of the analysis in the forgoing section, it can be seen that the $\mathrm{THD}_{\mathrm{u}}$ value on ALG - ALG/L at $02: 00 \mathrm{Hrs}$ in the first case was $5.26 \%$ at a total cascaded harmonic voltage value of $18.5 \mathrm{kV}$. On ALG -
FOW Distribution Line at the same time, the $\mathrm{THD}_{\mathrm{u}}$ value was estimated to be $20.4 \%$.

Table 6: Average Network THDu

\begin{tabular}{|l|c|c|c|c|c|}
\hline \multirow{2}{*}{$\begin{array}{c}\text { Distribution } \\
\text { Line }\end{array}$} & \multicolumn{3}{|c|}{ THD records (\%) } & Average THD $_{\mathrm{u}}$ \\
\cline { 2 - 5 } & $02: 00$ & $09: 00$ & $21: 00$ & $23: 00$ & $(\%)$ \\
\hline ALG - ALG/L & 5.26 & 5.03 & - & - & 5.145 \\
ALG - FOW & 20.40 & 21.40 & 18.50 & - & 20.100 \\
ALG - ADM & - & 30.00 & 23.77 & - & 26.885 \\
ALG - ANI & - & 31.90 & 28.40 & 18.50 & 26.266 \\
ADM - ANI & - & 20.70 & - & - & 20.700 \\
ALG - BAN & - & 14.80 & - & - & 14.800 \\
\hline
\end{tabular}

At 09:00Hrs, the $\mathrm{THD}_{\mathrm{u}}$ values on ALG - ADM Distribution Line, ALG - ALG/L Distribution Line, ALG - ANI Distribution Line, ALG - FOW Distribution Line, ADM - ANI Distribution Lineand ALG BAN/IDistribution Line were calculated to be $30 \%$, $5.03 \%, 31.9 \%, 21.4 \%, 20.7 \%$ and $14.8 \%$ respectively. At $21: 00 \mathrm{Hrs}$, the value of $\mathrm{THD}_{\mathrm{u}}$ for the case of ALG ADM Distribution Linewas 23.77\%; for the case of ALG - ANI Distribution Line, $\mathrm{THD}_{\mathrm{u}}$ was estimated to be $28.4 \%$, whereas, for the case of ALG - FOW Distribution Line, the value was calculated to be $18.5 \%$.

Finally, at 23:00Hrs, the value of ALG - ANI Distribution Linewas also estimated to be $18.5 \%$.

\section{CONCLUSION}

This paper has presented fundamentals of harmonic studies and also reviewed issues related to the concept. The results of power flow and its harmonic analysis of the Distribution Lines obtained from a research study were made use in this paper. However, parts of the result of harmonic analysis are presented as harmonics with corresponding impedances. Hence, the $\mathrm{THD}_{\mathrm{u}}$ of the Distribution Lineswere computed using the harmonic content parameters of the waveform, i.e. harmonic voltage and impedance magnitude values. The results of these $\mathrm{THD}_{\mathrm{u}}$ for the Distribution Lines are also presented. Network average $\mathrm{THD}_{\mathrm{u}}$ was evaluated, while a summary discussion of these $\mathrm{THD}_{\mathrm{u}}$ values of the lines was presented.

\section{REFERENCES}

[1] Belgin, T. and Kaypmaz, T.C. "Harmonic Measurements and Power Factor Correction in a Cement Factory", International Conference on 
Electrical and Electronics Engineering, Istanbul, ELECO'99, 1999, pp. 199-202.

[2] Owen, E.L. "A History of Harmonics in Power Systems", IEEE Industry Applications Magazines, Vol. 4, no. 1, 1998, pp. 6-12.

[3] Mansoor, A., et al. "Predicting the net Harmonic currents produced by Large Numbers of Distributed single-phase Computer Loads" IEEE Transactions on Power Delivery, Vol. 10, no. 4, 1995, pp. 2001-2006.

[4] Wu, C. and Chan, Y., "Harmonic Analysis and Mitigation of a power System with Specially Connected Transformers", Latest Advances in Science and Computational Intelligence pp. 182 185, ISBN: 9781618040947.

[5] Buso, S., Malesani, L., Mattavelli, P. and Veronese, R., "Design and Fully Digital Control of Parallel Active Power Filters for Thyristor Rectifiers to Comply with IEC-1000-3-2 Standards, IEEE Transactions on Industry Applications, vol. 34, 1998, pp. 508-517.

[6] Czarnecki, L.S., "An Overview of Methods of Harmonic Suppression in Distribution Systems" in Proceedings of the IEEE Power Engineering Society Summer Meeting, vol. 2, 2000, pp. 800-805.

[7] Ekrem, G., Independent Component Analysis for Harmonic Source Identification in Electric Power Systems. Electrical Engineering Department, Drexel University. Philadelphia, 2007, pp. 9-30, PhD Thesis.

[8] Mazumdar, J.,System and Method for Determining Harmonic Contributions from Non-linear Loads in Power System, Electrical and Computer Engineering, Georgia Institute of Technology, Atlanta, 2006. pp. 15-42, 165-177, PhD Thesis.

[9] Siemens.Harmonics in Power Systems - Causes, Effects and Control. Georgia : Siemens Industry, Inc., 2013, pp. 10-16.
[10] Heydt, G. and Risal, A.Apparatus and Method to Identify Harmonic Producing Loads., United States of America, April 16, 1996, pp. 529-539.

[11] Fauri, M. and Ribeiro, P. "A Novel Approach to Nonlinear Load Modelling", Proceedings of the 6th International Conference on Harmonics in Power Systems, Bologna, Italy, 1996, pp. 201-205.

[12] IEEE Standard 519-1992 "IEEE Recommended Practices and Requirements for Harmonic Control in Electric power Systems". USA, 1993.

[13] Hoevenarrs, T., LeDoux, K. and Colosino, M. "Interpreting IEEE STD 519 and Meeting its Harmonic Limits in VFD Applications" Proceedings of the IEEE Industry Applications Society 50th Annual Petroleum and Chemical Industry Conference, September 15-17, 2003, pp. 145-150.

[14] Halpin, S.M., "Overview of Revisions to IEEE Standard 519-1992", Proceedings of the IEEE International Symposium on Quality and Security of Electric Power Delivery Systems CIGRE/PES, 2003, pp. 65-68.

[15] Rafiei, S.M. and Ghazi, R. and Toliyat, H., "IEEE-519Based Real-time and Optimal Control of Active Filters Under Nonsinusoidal Line Voltages Using Neutral Networks", IEEE Transactions on Power Delivery, Vol. 17, 2002, pp. 815-821.

[16] Ludbrook, A., "IEEE 519-Harmonic Current Goals and Diversity - A Proposal" Proceedings of the International Conference on Harmonics and Quality of Power (ICHQP98), 2001, pp. 797-800.

[17] Kumar, Bhunesh.,Design of Harmonic Filters for Renewable Energy Applications. Department of Wind Energy, Gotland University, Visby, Sweden, 2011. PhD Thesis.

[18] Adesina, L.M. and Fakolujo, O.A., "Harmonic Analysis in a $33 \mathrm{kV}$ Distribution Network: A Case Study of Island Business District" IEEE African Journal of Computing and ICTs, Vol. 8, No.2, June 2015. 